\title{
Primary Thyroid Lymphoma Presenting in Stridor
}

\author{
Tulasi Kota Karanth', Ajay M. Bhandarkar', Kailesh Pujary ${ }^{1}$, Vidya Monappa ${ }^{2}$
}

Case

Report

\author{
${ }^{1}$ Department of Otorhinolaryngology, head and neck surgery, Kasturba Medical College and \\ Hospital, Manipal Academy of Higher Education, Manipal, Karnataka, INDIA.
}

${ }^{2}$ Department of Pathology, Kasturba Medical College and Hospital, Manipal Academy of Higher Education, Manipal, Karnataka, INDIA.

\begin{abstract}
Primary thyroid lymphoma is an uncommon disorder of the thyroid gland. It is an aggressive yet potentially curable malignancy when diagnosed early and treated appropriately. We present three cases who presented with rapidly enlarging neck swelling of approximately one month duration and stridor. Contrast enhanced computed tomography of neck showed enlarged thyroid gland compressing trachea and displacing major vessels. All three patients underwent isthmusectomy and tracheostomy under general anaesthesia. Histopathology showed features of diffuse large B cell lymphoma. They were planned to receive chemo-radiation. Two became seriously ill and one was lost to follow-up. Therefore, early consideration should be given to the possibility of primary thyroid lymphoma in patients presenting with rapidly enlarging neck swelling and stridor. Contrast enhanced computed tomography provides quick useful information to plan for surgical management of airway and immunohistochemistry of tissue provides key information in making the diagnosis. Prognosis is grave when the patient presents late.
\end{abstract}

Key Words: Diffuse large B-cell lymphoma, stridor, thyroid lymphoma, tracheostomy.

Received: $31^{\text {th }}$ May 2019, Accepted: $13^{\text {th }}$ October 2019

Corresponding Author: Tulasi Kota Karanth, Department of Otorhinolaryngology, head and neck surgery, Kasturba Medical College and Hospital, Manipal Academy of Higher Education, Manipal, Karnataka, INDIA 576104, Tel.: +91 820-2922218, E-mail: karanthtk@gmail.com.

ISSN: 2090-0740, March 2020 Vol. 21, No. 1.

\section{INTRODUCTION}

Primary thyroid lymphoma (PTL) is an uncommon disorder of the thyroid gland. It is an aggressive yet potentially curable malignancy when diagnosed early and treated appropriately ${ }^{[1,2]}$. It is usually seen in elderly females as a rapidly enlarging neck mass. Pressure over adjacent neck structures cause dysphagia, dyspnoea, superior vena cava syndrome and choking in approximately $30-50 \%$ or patients ${ }^{[3]}$. There have been 13 cases of PTL presenting with stridor in the literature. Ten of these patients required surgery to secure the airway ${ }^{[4]}$. We discuss three cases of PTL who presented with stridor in our institution.

\section{CASE REPORT}

\subsection{Case 1}

A 67-year-old lady with hypothyroidism presented to clinic with rapidly enlarging neck mass of one month duration and stridor. On examination, a large swelling was seen over neck occupying the entire right side of neck displacing laryngo-tracheal framework and carotid to left. Flexible endoscopy revealed a displaced and compressed larynx with two millimetre glottic chink. Contrast enhanced computed tomography (CECT) of neck revealed an enlarged thyroid gland extending between the second cervical vertebra and first thoracic vertebra, infiltrating sternocleidomastoid and strap muscles with postero-lateral displacement of carotid arteries and internal jugular veins (Figure 1). As her stridor worsened, she underwent emergency isthmusectomy and tracheostomy after awake fibre-optic naso-tracheal intubation. After placing a Kocher's incision and elevating sub-platysmal planes, gland was delineated from larynx superiorlry. Dissection was performed from superior to inferior till trachea was reached a tracheostomy could be performed. Retrosternal tumor was not addressed during the procedure. Hemostasis was achieved by using bipolar cautery and Surgicel ${ }^{\circledR} \quad$ (Ethicon, Switzerland). Intra-operatively superior laryngeal or recurrent laryngeal nerves coculd not be identified. Post-operatively she developed aspiration due to injury to superior and recurrent laryngeal nerves, which was managed conservatively with nasogastric tube feeding. Histopathological examination of thyroid tissue revealed sheets of monomorphous lymphoid cells with irregular nuclear membrane and numerous mitotic figures. Surrounding tissue showed desmoplastic stroma with lymphoplasmacytic infiltration. Immuno-histochemical staining was performed and positive staining was seen for 
CD 20, CD 3, CD 10, BCL 6, MUM1 and Ki67. Staining revealed presence of CD-20, MUM1 antigen and $80 \%$ positivity for Ki67. She was diagnosed to have diffuse large B-cell lymphoma of thyroid. Pre-treatment evaluation included bone marrow biopsy and positron emission tomography (PET) - CT. Investigations revealed localised disease. She was planned to receive pre-phase treatment with prednisolone, rituximab and cyclophosphamide followed by six cycles of rituximab, cyclophosphamide, doxorubicin, vincristine, prednisolone (R-CHOP) regimen. After five days of treatment, she became unresponsive and was shifted to receive intensive care. She was diagnosed to have sepsis due to possible aspiration pneumonia and she passed away after attempts of resuscitation.

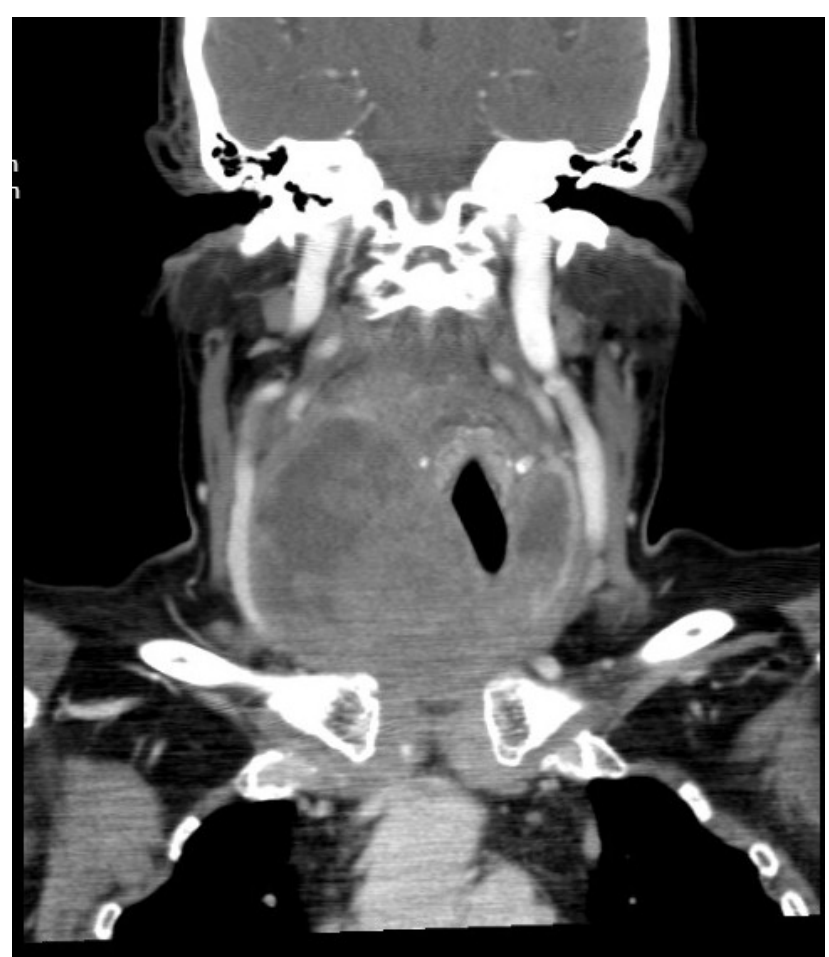

Fig. 1: Contrast-enhanced CT image of neck in coronal plane. Image describes a multiple ill-defined heterogeneously hypoenhancing hypodense lesions in an enlarged thyroid gland that has displaced carotid artery and internal jugular veins laterally.

\subsection{Case 2}

A 46-year-old man presented to the out-patient clinic with rapidly enlarging swelling of one month duration. On examination, he was stridorous with neck swelling spanning entire neck with no palpable landmark. Flexible endoscopy of the larynx revealed a narrowed airway and aspiration of saliva. Contrast enhanced CT of the neck showed an ill-defined minimally enhancing homogenous soft tissue density involving bilateral submandibular, masticator, retropharyngeal, prevertebral and paraspinal spaces, extending inferiorly into mediastinum (Figure 2). Fat planes were lost with adjacent neck structures. He was taken up for debulking, emergency tracheostomy and biopsy of tumour. A four centimetre long vertical incision was placed over midline reaching supra sternal notch inferiorly.
Dissection was performed within the tumor, deep into the tissue till trachea was reached. A window was created over tracheal rings and an endotracheal tube was placed through the tracheostoma. Hemostasis was achieved from bleeding tumor by bipolar cautery and placement of Surgicel ${ }^{\circledR}$ (Ethicon, Switzerland). Tumor bits excised during the procedure was sent for histopathological examination. Histopathological examination revealed sheets of large lymphoid cells with high nucleus to cytoplasm ratio, scant cytoplasm, pleomorphic hyperchromatic round nuclei, coarse chromatin with prominent nucleoli and frequent mitosis. Immuno-histochemical staining was performed and positive staining was seen for CD 20, CD 3, CD 10, Bcl 6, Bcl 2 and Ki67. Tumour was immuno-histochemically positive for CD-20, Bcl-2 and 50\% positive for Ki67. He was diagnosed to have non-Hodgkin lymphoma - diffuse large B cell type. He was advised definitive chemotherapy. He was not willing for treatment and was further lost to follow up.

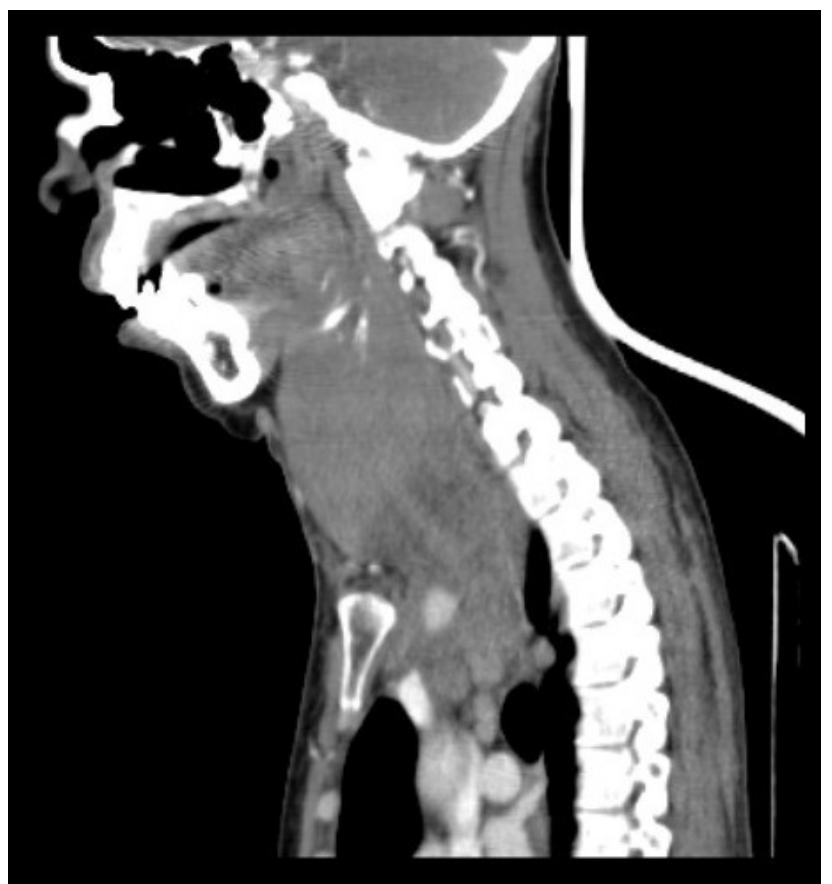

Fig. 2: Contrast-enhanced CT image of neck in sagittal plane. Image describes an ill-defined minimally enhancing homogenous soft tissue density involving bilateral pharyngeal mucosa, retropharyngeal, perivertebral and para spinal spaces. Fat planes are lost with adjacent structures of the neck.

\subsection{Case 3}

A 36-year old man presented to the out-patient clinic with complains of rapidly increasing neck swelling of one month duration. On examination, a large swelling was seen at the region of thyroid which was hard and extending retrosternally. He was comfortable with no signs of distress or stridor. Flexible endoscopy of larynx showed normal laryngeal anatomy. Fine needle aspiration was performed from the swelling which suggested poorly differentiated carcinoma. Following the initial evaluation, he was lost to follow up and hence the possible diagnosis could not be communicated. He presented a month later 
to emergency with difficulty breathing. Contrast enhanced CT showed a large well defined heterogeneously enhancing lesion in the region of thyroid extending between fourth cervical vertebra and third thoracic vertebra. Mass was compressing the larynx and trachea. Common carotid arteries were pushed postero-laterally and internal jugular vein was thrombosed. Enlarged lymph nodes were seen in bilateral level II, III, IV, left para-tracheal, para-aortic, subaortic and sub-carinal region. He underwent emergency total thyroidectomy and tracheostomy under general anaesthesia. Total thyroidectomy was performed following a Kocher's incision over the anterior neck. Infiltrated musculature, for example, the strap muscles, were dissected along with the main total thyroidectomy specimen. Thyroid was not fixed to larynx or trachea. Retrosternal tumor was addresssed with the help of extreme extension of neck during positioning and minimal dissection into the thorasic cavity. Sternotomy was not necessitated during the surgery. Histopathological examination of the thyroid tissue revealed diffuse proliferation of large lymphoid cells with enlarged nuclei, moderate amphophillic cytoplasm with brisk mitosis and focal areas of necrosis in the background of Hashimoto thyroiditis (Figure 3). Immunohistochemistry was performed and positive staining was seen for CD 20, CD 3, CD 10, Bcl 6, BCL 2, MUM1 and Ki67. Staining revealed strong positivity for CD 20 (Figure 4 ) and $\mathrm{Bcl} 2 ; 30 \%$ positivity for $\mathrm{CD} 10,20 \%$ for Bcl 6, 20\% for MUM1 and 70\% for Ki67. A diagnosis of large B cell lymphoma was considered. Pre-treatment evaluation included bone marrow biopsy and PET-CT. Investigations revealed localised disease. He was planned for pre-phase treatment with prednisolone, rituximab followed by six cycles of rituximab, cyclophosphamide, doxorubicin, vincristine, prednisolone (R-CHOP) regimen. During the course of treatment, he developed plaques over his face and neck. Biopsy from the skin lesion revealed metastasis. As the disease progressed when on R-CHOP, he was planned for salvage chemotherapy. He developed breathlessness and was shifted to intensive care unit. Total counts and procalcitonin were raised. He was diagnosed to have sepsis with septic shock. He refused further treatment and was discharged home.

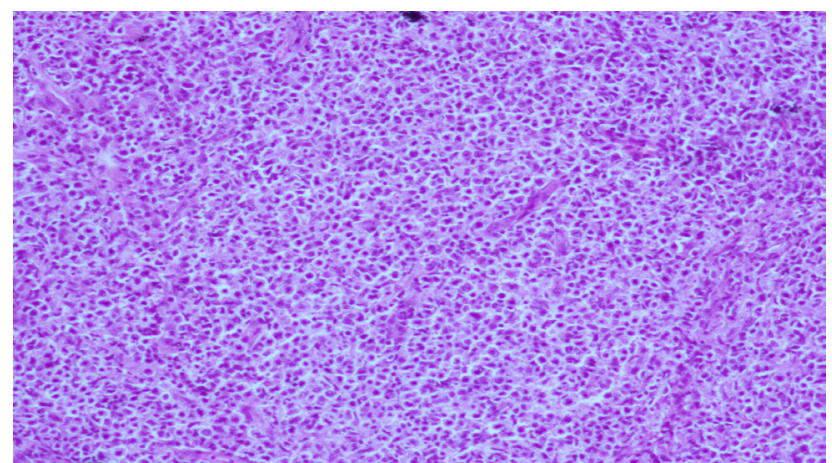

Fig. 3: Haematoxylin and eosin staining image. Image viewed under $10 \mathrm{x}$ magnification describes destruction of thyroid parenchyma by diffuse proliferation of large lymphoid cells with enlarged nuclei, one or more prominent nucleoli, moderate amphophillic cytoplasm with brisk mitosis and focal areas of necrosis.

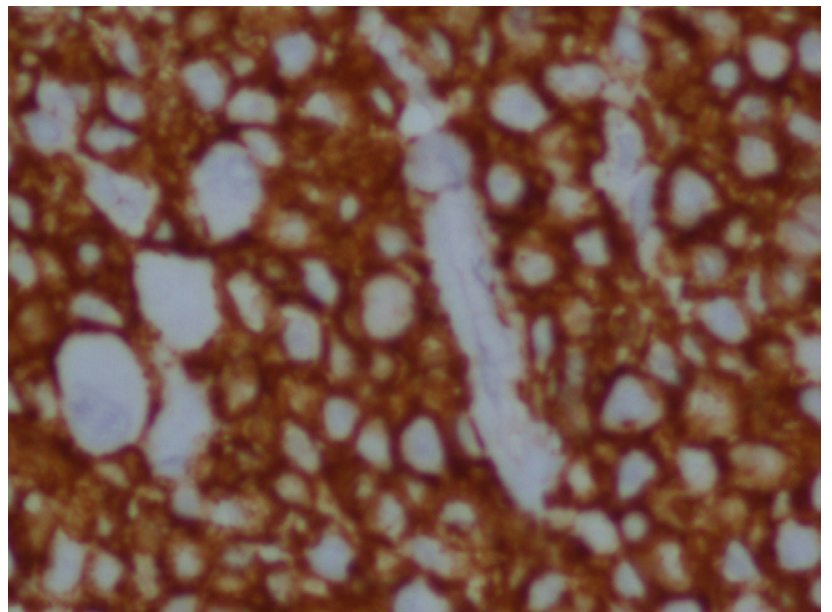

Fig. 4: Immunohistochemical staining image. Image viewed under 20x magnification describes strongly positive CD 20 response diffusely among lymphoid cells.

\section{DISCUSSION}

Primary thyroid lymphoma is a rare malignancy of the thyroid gland with an estimated annual incidence of two per million ${ }^{[5]}$. It is an uncommon disease seen more often in women ${ }^{[6]}$. in their sixth or seventh decade of life ${ }^{[7]}$. They usually complain of a rapidly enlarging neck swelling. One-third of them also complain of compressive symptoms in neck, such as dysphagia, dyspnoea, hoarseness and pain over thyroid ${ }^{[5]}$. It is extremely rare for patients with PTL to present with stridor.

Sinha et al in the year 2005 summarised 13 cases of PTL with stridor, described in the literature ${ }^{[4]}$. Nine of them required a surgical procedure to decompress trachea or secure the airway. Decompression was achieved by partial, subtotal, near total and total thyroidectomies. Tracheostomy was performed in four of them. Two required cardiopulmonary bypass during tracheostomy procedure ${ }^{[4]}$.

It was unusual to find two men in fourth to fifth decade of life to come with rapidly enlarging neck swelling of only one month duration with no previous history suggestive of thyroid disease to be diagnosed with PTL. All our patients were having stridor at presentation and required surgery to decompress and secure the airway.

Primary thyroid lymphoma is evaluated using ultrasound, CECT and fluorodeoxyglucose (FDG), PETCT scan to assess for the extent of disease ${ }^{[8]}$. Biopsy or fine needle aspirate cytology and immunohistochemical typing are essential to confirm the diagnosis and further classification $^{[8]}$. The most common form of PTL is diffuse large B-cell lymphoma (DLBCL), followed by mucosa associated lymphoid tissue (MALT) lymphoma. Other rare types include mixed type, follicular, small lymphocytic, Hodgkin, Burkitt, T-cell, Mantle cell and lymphoblastic lymphoma in decreasing order of incidence ${ }^{[9]}$. Immuno-histochemistry helps determine the lineage and developmental stage of lymphoma. Antibodies against CD-19, CD-20 identifies a B-cell lineage and CD-10 positivity identifies follicular lymphomas. Most 
DLBCLs show positive reaction to Bcl-6 and some to $\mathrm{Bcl}-2$. Presence of Immunoglobulin light chain and Bcl-2 differentiates MALT-lymphoma from others ${ }^{[8]}$.

Contrast enhanced CT is useful in evaluation of a patient with rapidly enlarging neck mass and stridor. It appears as a low attenuation mass in the region of thyroid compressing or infiltrating into surrounding tissue with little evidence of calcification or necrosis ${ }^{[10]}$. It helps us determine extent of the disease and altered anatomy in a short time ${ }^{[8]}$. Images taken can be re-assessed multiple times for careful planning of surgery.

The management of PTL, especially DLBCL includes six cycles of anthracyclin derived multi-drug chemotherapy. The commonly used drugs include rituximab, cyclophosphamide, doxorubicin, vincristine and prednisolone (R-CHOP). Radiation should be considered after chemotherapy in all patients unless they are aged less than 60 years and have no bad prognostic factors ${ }^{[11]}$. The overall survival of patients with thyroid non-Hodgkin's lymphoma ranges between $42 \%$ and $100 \%{ }^{[12]}$. In our series, all were planned to receive six cycles of R-CHOP. Two patients became severely ill requiring intensive care prior to completion of treatment and one was lost to follow-up.

\section{CONCLUSION}

We should consider the possibility of PTL in patients presenting with rapidly enlarging neck swelling and stridor. Contrast enhanced CT provides quick useful information to plan for surgical management of airway.

\section{CONFLICT OF INTEREST}

There are no conflict of interest.

\section{FUNDING}

This research did not receive any specific grant from funding agencies in the public, commercial, or not-forprofit sectors.

\section{REFERENCES}

1. Sakorafas GH, Kokkoris P, Harley DR. Primary thyroid lymphoma. Surgical Oncology 2010; 19 (4): e124-9.

2. Tsutsui K, Shibamoto Y, Yamabe H, Shima N, Dodo $\mathrm{Y}$, Ono $\mathrm{K}$, et al. A radiotherapeutic experience for localized extranodal non-Hodgkin's lymphoma: prognostic factors and re-evaluation of treatment modality. Radiother Oncol. 1991 Jun; 21(2):83-90.

3. Herranz-Antolin S, Castro-Martinez E, VinventeDelgado, Sastre-Marcos J, Anguirre-Sanchez Covisa M. Primary thyroid lymphomas. Experience in hospitals of Castilla-La. Endocrinol Diabetes Nutr. 2018 Mar; 65(3):184-6.

4. Sinha S, Aish L, Oo TH. Primary thyroid lymphoma presenting with stridor. Am J Clin Oncol. 2005 Oct; 28(5):531-3.

5. Pedersen RK, Pedersen NT. Primary non-Hodgkin's lymphoma of the thyroid gland: a population based study. Histopathology. 1996; 28:25-32.

6. Thieblemont C, Mayer A, Dumontet C, Barbier Y, Callet-Bauchu E, Felman P, et al. Primary thyroid lymphoma is a heterogeneous disease. J Clin Endocrinol Metab. 2002; 87:105-11.

7. Sarinah B, Hisham AN. Primary lymphoma of the thyroid: diagnostic and therapeutic considerations. Asian J Surg. 2010; 33:20-4.

8. Stein SA, Wartofsky L. Primary thyroid lymphoma: a clinical review J Clin Endocrinol Metab. 2013 Aug; 98(8):3131-8.

9. Graff-Baker A, Roman SA, Thomas DC, Udelsman R, Sosa JA. Prognosis of primary thyroid lymphoma: demographic, clinical, and pathologic predictors of survival in 1,408 cases. Surgery . 2009;146:1105-15.

10. Takashima S, Ikezoe J, Morimoto S, Arisawa J, Hamada S, Ikeda $\mathrm{H}$ et al. Primary thyroid lymphoma: evaluation with CT. Radiology. 1988 Sep;168(3): 765-8.

11. Alzouebi M, Goepel JR, Horsman JM, Hancock BW. Primary thyroid lymphoma: the 40 year experience of a UK lymphoma treatment center. Int J Oncol. 2012 Jun; 40(6):2075-80.

12. Mondal SK. Cytodiagnosis of primary thyroid lymphoma with histologic correlation: a case report. Diagn Cytopathol. 2012 May; 40(5):444-6. 